Original Paper http://ajol.info/index.php/ijbcs http://indexmedicus.afro.who.int

\title{
Évaluation de la contamination des eaux de la lagune Ebrié (Zones IV et V), Côte d'Ivoire en arsenic, plomb et cadmium: variations spatio-temporelles et risques sanitaires
}

\author{
Ayénan Marc Olivier TOGBE ${ }^{1,2 *}$, Kouamé Victor KOUAME ${ }^{2}$, Koffi Marcellin YAO ${ }^{3}$, \\ Ahmed Ahbeauriet OUATTARA ${ }^{4}$, Abiba Sanogo TIDOU ${ }^{5}$ et Boua Celestin ATSÉ ${ }^{3}$ \\ ${ }^{1}$ Direction de l'Aquaculture et des Pêches, BP V 19 Abidjan, Côte d'Ivoire. \\ ${ }^{2}$ Université NANGUI ABROGOUA, Abidjan, Côte d'Ivoire 02 BP 801 Abidjan, Côte d'Ivoire. \\ ${ }^{3}$ Centre de Recherches Océanologiques (CRO), BP V18 Abidjan, Côte d'Ivoire. \\ ${ }^{4}$ Université Félix HOUPHOUËT BOIGNY, UFR Sciences des Structures de la Matière et Technologie, \\ BP 582 Abidjan 22, Côte d'Ivoire. \\ ${ }^{5}$ Université Jean Lorougnon Guédé, UFR Environnement, BP 150 Daloa, Côte d'Ivoire. \\ *Auteur correspondant; E-mail : marctogbe@yahoo.fr
}

\section{RESUME}

Les secteurs IV et V de la lagune Ebrié subissent une pression anthropique de plus en plus importante du fait de l'augmentation de la population et de l'intensification des activités agroindustrielles. L'objectif de cette étude est d'évaluer le niveau de contamination et les risques sanitaires des éléments ( $\mathrm{As}, \mathrm{Cd}$ et $\mathrm{Pb})$ dans les eaux des secteurs IV et V de la lagune Ebrié. Au total, 156 échantillons d'eaux ont été prélevés mensuellement à 11 stations de décembre 2014 à novembre 2015. Les résultats ont montré qu'à l'exception de l'arsenic, les concentrations moyennes en plomb $(6,16 \pm 6,57 \mu \mathrm{g} / \mathrm{L})$ et en cadmium $(10,97 \pm 9,77 \mu \mathrm{g} / \mathrm{L})$ ont été supérieures à la valeur chronique des critères canadiens pour la vie aquatiques. Les valeurs de la dose journalière chronique (DJE) pour le plomb, le cadmium et l'arsenic ont varié respectivement de 0,0001 à 0,029 , de 0,00005 à 0,009 et de 0,000084 à $0,017 \mu \mathrm{g} / \mathrm{kg} / \mathrm{j}$. Cependant, les valeurs de l'indice du risque potentiel non cancérigène ou quotient de danger (HQ) et de l'indice de risque (HI) ont été inférieures à 1; ce qui traduit que les concentrations en cadmium, arsenic et plomb ne présentent aucun danger pour la santé humaine dans la zone d'étude.

(C) 2019 International Formulae Group. All rights reserved

Mots clés : Lagune Ebrié, éléments traces métalliques, pollution métallique.

\section{Assessment of Ebrie Lagoon waters contamination (Sectors IV and V), Côte d'Ivoire, in arsenic, lead and cadmium: Spatio-temporal variations and sanitary risks}

\begin{abstract}
Sectors IV and V of Ebrié Lagoon are facing significant anthropogenic pressures due to recent increase in human population and agro-industrial activities. The objective of the present study was to assess trace element $\mathrm{As}, \mathrm{Cd}$ and $\mathrm{Pb}$ concentration levels in Ebrie Lagoon waters, especially in sectors IV and V along with their spatial and seasonal variations, and associated human health risks relative to dermal absorption. A
\end{abstract}


total of 156 water samples were collected at eleven stations from December 2014 to November 2015. The results showed that the average lead concentration $(6.16 \pm 6.57 \mu \mathrm{g} / \mathrm{L})$ was above the chronic value and lower than the acute value, the average arsenic concentration $(7.30 \pm 14,14 \mu \mathrm{g} / \mathrm{L})$ was below the aquatic life criteria set by the Canadian Ministry of Sustainable Development, the Environment and the Parks (MDDEP), while the average cadmium concentration $(10.97 \pm 9.77 \mu \mathrm{g} / \mathrm{L})$ was higher than the aquatic life criteria. The Chronic Daily Intake (DJE) values ranged from 0.0001 to $0.029,0.00005$ to 0.009 , and 0.000084 to $0.017 \mu \mathrm{g} / \mathrm{kg}$ for lead, cadmium and arsenic, respectively. The Hazard Quotient (HQ) and the Hazard Index (HI) were found to be less than 1 at all the stations during all the seasons, showing no health risks to human.

(C) 2019 International Formulae Group. All rights reserved

Keywords: Ebrié Lagoon, Metal Trace elements, Metal Pollution.

\section{INTRODUCTION}

La pollution des milieux aquatiques par les éléments traces métalliques (ETM) est devenue un problème majeur dans le monde à cause de l'intensification des activités industrielles et l'urbanisation rapide. Cette pollution métallique provoque une dégradation de la qualité des eaux et menace la vie des organismes aquatiques et celles des humains. En effet, les ETM sont particulièrement préoccupants à cause de leurs propriétés non biodégradables et des effets toxiques qu'ils peuvent engendrer lorsqu'ils sont accumulés à des concentrations élevées dans les organismes (Sundaray et al., 2006; Ahmad et al., 2010 ; Ouattara et al., 2018).

Les lagunes sont les écosystèmes marins les plus productifs du monde en raison de leur emplacement entre terre et mer. Elles servent de pépinières et d'aires d'alimentation de nombreuses espèces continentales et marines. La plupart des lagunes sont caractérisées comme des écosystèmes complexes avec un équilibre fragile car les lagunes reçoivent les eaux continentales et marines (Hu et al., 2018). Les ETM sont introduits dans ces lagunes à travers des sources naturelles (l'érosion des terres, les activités volcaniques et les dépôts atmosphériques) et les sources anthropiques (Kouassi et al., 2015 ; Hu et al., 2018). Les principales sources anthropiques sont l'utilisation des engrais et des pesticides dans l'agriculture, les rejets urbains et industriels (Kouassi et al., 2015 ; Burgos-Nunez et al., 2017). Les fleuves et les rivières sont également des sources d'ETM dans les eaux lagunaires. Dans les eaux lagunaires, les ETM existent en général sous plusieurs formes (dissoutes, phases particulaires et sédimentaires). Les sédiments représentent les principaux puits des ETM et agissent comme des sources secondaires potentielles (Zhang et al., 2017 ; Hu et al., 2018). Les ETM piégés dans les sédiments peuvent se retrouver dans la colonne d'eau lors de variation des paramètres physico-chimiques et géologiques du milieu. Ainsi leur comportement dans les eaux lagunaires est très complexe et dépend de leurs formes physico-chimiques spécifiques et des caractéristiques du milieu. Les ETM peuvent également contaminer la chaîne alimentaire par bioaccumulation et provoquer des risques pour les organismes vivants ainsi que pour les humains (Wei et al., 2016 ; Ouattara et al., 2018 ). De ce fait, l'évaluation de la contamination des lagunes par les ETM est d'une importance capitale pour prévenir les risques écologiques.

La lagune Ebrié est le système lagunaire le plus important de l'Afrique de l'Ouest. Plusieurs études ont rapporté dans la littérature une contamination métallique importante de la zone urbaine de la lagune Ebrié (Yao et al., 2009; Adama et al., 2012; Tuo et al., 2013; Kinimo et al., 2018). Cependant, peu d'informations sont disponibles sur les contaminations en ETM des eaux lagunaires en dehors de la zone urbaine. Cette étude se focalise sur la zone rurale ouest de la lagune Ebrié où l'urbanisation tend à s'intensifier et l'agriculture et les activités industrielles se développent de plus en plus. Les engrais et pesticides sont couramment utilisés dans les grandes exploitations agro industrielles et les 
activités off-shore sont également présentes dans la zone (Rauch et Pacyna, 2009 ; FOXTROT, 2012). L'objectif de cette étude est d'évaluer le niveau de contamination métallique de la zone rurale ouest de la lagune Ebrié. Pour ce faire, les concentrations des eaux lagunaires en arsenic, plomb et cadmium ont été mesurées mensuellement au cours d'une année hydrologique, les variations spatio-temporelles ont été examinées et les risques écologiques ont été évalués par le calcul de l'indice du risque non cancérigène.

\section{MATERIELS ET METHODES \\ Présentation des sites d'étude}

Le système lagunaire Ebrié s'étire sur $120 \mathrm{~km}$ et sa largeur ne dépasse pas $7 \mathrm{~km}$. Il comprend la lagune Ebrié proprement dite et les lagunes Aghien et Potou. La lagune Ebrié est la plus vaste des lagunes ivoiriennes avec une superficie de $566 \mathrm{~km}^{2}$. Le volume de cette lagune est environ de 2,5 milliards $\mathrm{de}^{3}$. La profondeur moyenne est de 4,8 $\mathrm{m}$ et quelques fosses proches d'Abidjan dépassent $20 \mathrm{~m}$ (port, baie d'Abou-Abou, etc.). La lagune Ebrié est séparée de l'Océan Atlantique par un cordon littoral sableux de 1 à $8 \mathrm{~km}$ traversé en sa partie centrale par le canal de Vridi depuis 1951. Ce canal, large de $300 \mathrm{~m}$ et profond d'environ une vingtaine de mètres est la seule voie de communication avec la mer (Dufour et al., 1994).

Des critères aussi bien morphologiques que biologiques ont permis l'identification de six principaux secteurs dans la lagune Ebrié. Cette classification tient en effet compte du taux de renouvellement annuel des eaux selon les zones considérées (Durand et Guiral, 1994):

- Secteur I: composé des lagunes Potou et Aghien;

- Secteur II: s'étend de Bassam à Abidjan;

- Secteur III: présent dans la région d'Abidjan;

- Secteur IV: d'Abidjan à l'Agnéby;

- Secteur V: de l'Agnéby à $15 \mathrm{~km}$ du canal d'Assagny;

- Secteur VI: de l'extrémité occidentale avant le canal d'Assagny.
Les sites retenus pour cette étude sont les secteurs IV et V (Figure 1)

Le plan d'échantillonnage a été conçu de sorte que les stations de prélèvement soient représentatives de la zone étudiée (conditions générales en amont, au centre, et en aval). Onze (11) stations de prélèvements ont été retenues dont les noms et les caractéristiques sont présentées dans le Tableau 1.

\section{Prélèvement des échantillons et méthodes d'analyse}

L'échantillonnage des eaux de lagune a été effectué de décembre 2014 à novembre 2015. La période d'échantillonnage s'étend sur quatre saisons climatiques dont la grande saison sèche (décembre à mars), la grande saison des pluies (d'avril à juillet), la petite saison sèche (août à septembre), la petite saison des pluies (octobre à novembre). Les échantillons ont été collectés à onze (11) stations, six stations du côté du département de Dabou et cinq (5) stations dans le département de Jacqueville. Les échantillons d'eau ont été prélevés à l'aide d'une bouteille Niskin et recueillis dans les flacons en polyéthylène tout en prenant soin de les rincer trois fois avec l'échantillon. Trois échantillons ont été prélevés par station. Les flacons ont été conservés immédiatement au frais $\left(4^{\circ} \mathrm{C}\right)$ et à l'abri de la lumière, dans une glacière sur le terrain pour éviter toute réaction photochimique ou biologique. Au laboratoire, tous les échantillons d'eau ont été directement conservés dans des congélateurs à des températures inférieures à $4{ }^{\circ} \mathrm{C}$. Les échantillons d'eau ont été filtrés à travers des papiers filtres Whatmann GFC de $0,45 \mu \mathrm{m}$, acidifiés avec de l'acide nitrique $(2 \%)$ et stockés dans des bouteilles en polyéthylène jusqu'à l'analyse selon la procédure standard (APHA, 1998). Le dosage de ces métaux Cd, $\mathrm{Pb}$, et As a été effectué par absorption atomique (SpectrAA100 Varian spectrophotometer, Japan). Chaque échantillon a été analysé en triple. Les limites de détection des éléments traces métalliques ont été de $\mathrm{Cd}(0,01 \mu \mathrm{g} / \mathrm{L}), \mathrm{Pb}(0,06 \mu \mathrm{g} / \mathrm{L})$, As $(0,05 \mu \mathrm{g} / \mathrm{L})$. 


\section{Calcul des indices de pollution des eaux Évaluation du risque non cancérigène}

Le risque est défini comme le processus d'estimation d'apparition d'un évènement et la probabilité d'avoir des effets néfastes sur la santé de l'Homme pendant un temps ou période donné. L'évaluation du risque sur la santé de l'Homme se fait suivant quatre étapes dont l'identification du risque, l'évaluation de la toxicité (dose-réponse), l'exposition et la caractérisation du risque. L'estimation de la dose ou quantité prise par un être humain afin d'être contaminé est déterminée par la Dose Journalière Chronique (DJE) ou CDI (Chronic Daily Intake de l'Anglais). Dans cette étude seule la voie dermique a été considérée.

La DJE détermine la quantité de substance chimique absorbée par la peau par kilogramme de la masse par personne par jour $(\mathrm{mg} / \mathrm{kg} / \mathrm{j})$ selon l'équation (1) suivante:

\section{$\mathrm{DJE}=\frac{{\mathrm{CXSA} \times \mathrm{K}_{\mathrm{P}} \times \mathrm{ET} \times \mathrm{EF} \times \mathrm{ED} \times \mathrm{CF}}_{\text {BWXAT }}}{1)}$}

Où DJE désigne la dose journalière chronique exprimée en $(\mathrm{mg} / \mathrm{kg} / \mathrm{j}), \quad \mathrm{SA}$ représente la surface exposée exprimée $\left(\mathrm{cm}^{2}\right)$, $\mathrm{Kp}$ désigne le coefficient de perméabilité dermatologique exprimé en $(\mathrm{Cm} / \mathrm{h})$, ET représente le temps d'exposition exprimé en( $\mathrm{h} / \mathrm{j}$ ), EF représente la fréquence d'exposition exprimé en (j/an) ED représente la durée d'exposition exprimée en année, CF représente le facteur conversion exprimé en $\left(\mathrm{L} / \mathrm{cm}^{3}\right)$, BW désigne le poids par personne exprimé en $(\mathrm{kg})$, AT représente le temps moyen ou minimal qui s'exprime en jours (j).

Le Tableau 2 donne les valeurs des différents paramètres pour le calcul des risques. La caractérisation du risque se fait séparément selon les effets cancérigènes et non cancérigènes et inclue la discussion des facteurs qui résultent de la surestimation et de la sous-estimation du risque.

\section{Risque non cancérigène}

Le risque potentiel non cancérigène d'exposition aux contaminants (HQ) est évalué par le rapport des doses d'exposition aux contaminants calculées selon les voies d'exposition (DJE) (ingestion, dermatologique, inhalation) sur la dose de référence $(\mathrm{RfD})$ :

$$
\mathrm{HQ}=\mathrm{DJE} / \mathrm{RfD} \text { (2) }
$$

HQ est le quotient de danger. Cet indice donne une indication du niveau de risque de santé dû à une exposition à un polluant donné. DJE est la dose journalière chronique $(\mu \mathrm{g} / \mathrm{kg} / \mathrm{j})$ et $\mathrm{RfD}(\mathrm{mg} / \mathrm{kg} / \mathrm{j})$ est la dose journalière de référence d'un polluant qui ne cause aucun risque de santé à une population humaine exposée durant toute une vie. Lorsque HQ est supérieur à 1 (unité), les populations exposées aux polluants peuvent avoir des problèmes de santé. Pour déterminer l'ensemble des effets potentiels non cancérigène dû à plus d'un paramètre chimique, les valeurs de QD pour chaque élément chimique sont additionnées et exprimées par HI.

$$
H I=\sum_{i=1}^{n} H Q_{i}
$$

Si HI est inférieur à $1(\mathrm{HI}<1)$, alors il n'y a pas de risque de contamination chronique pour ce site; Si HI est supérieur à 1 comme conséquence de la somme des HQ, il serait approprié de prendre les HQ de chaque métal séparément.

\section{Analyses Statistiques}

Dans cette étude, toutes les analyses statistiques ont été réalisées par le logiciel Statistica 7.1. La variabilité spatiale et saisonnière des concentrations en éléments traces métalliques a été analysée par l'analyse des variances (ANOVA) à $(\mathrm{P}<0,05)$. La comparaison multiple des moyennes des concentrations en polluants métalliques a été effectuée au moyen du test de Kruskal-Wallis chaque fois que l'ANOVA a indiqué des différences significatives. Les valeurs moyennes sont présentées sous forme de moyenne \pm écart type. 


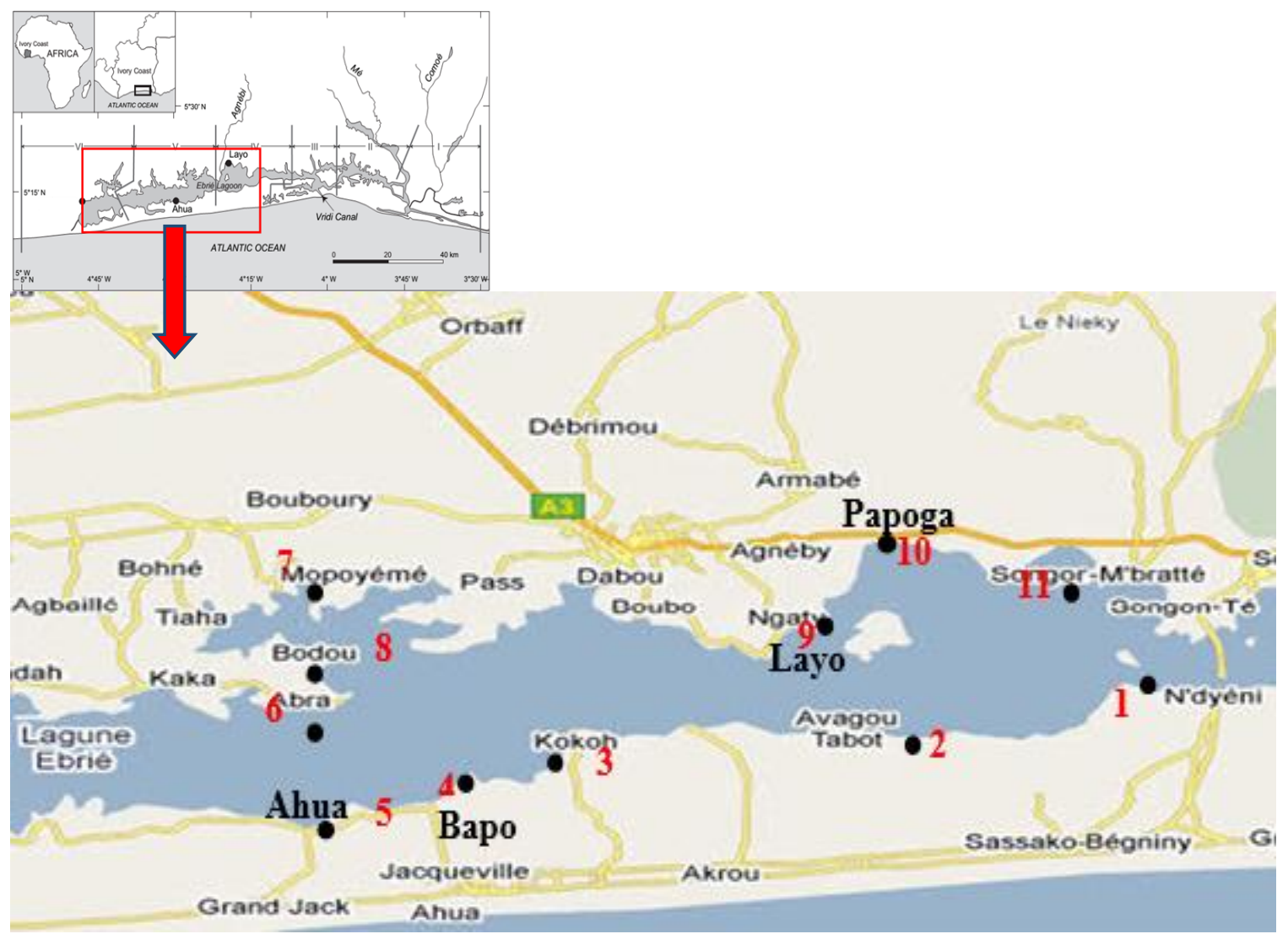

Figure 1 : Situation géographique des sites et stations d'échantillonnage.

Tableau 1: Désignation et caractéristiques des stations de prélèvement.

\begin{tabular}{|c|c|c|c|}
\hline Secteur & Code & Nom & Caractéristiques \\
\hline \multirow{6}{*}{ IV } & $\mathrm{La}$ & Layo & \multirow{6}{*}{$\begin{array}{l}\text { Zones } \\
\text { agricole, } \\
\text { industrielle } \\
\text { pêche. } \\
\text { Reçoit les } \\
\text { l'Agneby. } \\
\text { Superficie : } \\
\mathrm{km}^{2}\end{array}$} \\
\hline & $\mathrm{Nd}$ & Ndjem & \\
\hline & $\mathrm{Pa}$ & Papoga & \\
\hline & & & \\
\hline & So & Songon & \\
\hline & $\mathrm{Ta}$ & Taboth & \\
\hline & $\mathrm{Ah}$ & Ahua & Zones \\
\hline & $\mathrm{Ab}$ & Abraco & agricole, \\
\hline $\mathbf{V}$ & $\mathrm{Ba}$ & Bapo & industrielle et de \\
\hline & Ko & Koko & $\begin{array}{l}\text { peche. } \\
\text { Superficie: }\end{array}$ \\
\hline & $\mathrm{Gb}$ & Gbougbo & $\mathrm{km}^{2}$ \\
\hline & Mo & Mopoyem & \\
\hline
\end{tabular}


Tableau 2 : Paramètres caractérisant les valeurs de la dose journalière chronique (DJE).

\begin{tabular}{lccl}
\hline \multicolumn{1}{c}{ Paramètres } & Unité & Valeur & \multicolumn{1}{c}{ Source } \\
\hline Concentration du métal dans l'eau (C) & $\mu \mathrm{g} / \mathrm{L}$ & - & Présente étude \\
Fréquence d'exposition (EF) & $\mathrm{j} / \mathrm{an}$ & 365 & US EPA, 2013 \\
Durée d'exposition (ED) & an & 51 & World Bank (2014) \\
Masse corporelle (BW) & $\mathrm{kg}$ & 60,7 & Sangaré et al., 2016 \\
Espérance de Vie (AT) & jours & 10,95 & US EPA, 2013 \\
Surface de la peau exposé (SA) & $\mathrm{cm}^{2}$ & 18 & US EPA, 2013 \\
Temps d'exposition (ET) & $\mathrm{h} / \mathrm{j}$ & 0,6 & US EPA, 2013 \\
Facteur de conversion (CF) & $\mathrm{L} / \mathrm{cm}^{3}$ & 0,001 & US EPA, 2013 \\
Coefficient de perméabilité dermique (Kp) & & & \\
$\mathrm{Zn}$ & & 0,0006 & US EPA, 2013 \\
$\mathrm{Cu}$ & & 0,001 & US EPA, 2013 \\
$\mathrm{As}$ & $\mathrm{cm} / \mathrm{h}$ & 0,001 & US EPA, 2013 \\
$\mathrm{Cd}$ & & 0,001 & US EPA, 2013 \\
$\mathrm{Pb}$ & & 0,004 & US EPA, 2013 \\
\hline
\end{tabular}

\section{RESULTATS}

Variations spatio-temporelles de la contamination métallique des eaux de la lagune.

Les concentrations en plomb, en cadmium et en arsenic se situent entre 0,16 $35,45,0,3-49,59$ et $0,49-96,31 \mu \mathrm{g} / \mathrm{L}$ respectivement sur les onze sites d'échantillonnage (Tableau 3). Les valeurs moyennes des éléments traces métalliques sont $(6,16 \pm 6,57) \mu \mathrm{g} / \mathrm{L},(10,97 \pm 9,77) \mu \mathrm{g} / \mathrm{L}$ et $(7,30 \pm 14,14) \mu g / L$ pour le plomb, le cadmium et l'arsenic respectivement. Les concentrations du plomb et du cadmium sont plus élevées dans les eaux des stations du secteur IV de la lagune (Ahua, Abraco, Mopoyem, Koko, Bougbo) comparées à celles des stations du secteur V (Taboth, Papoga, Layo, Bapo, Songon, Ndjem). Les concentrations moyennes du plomb sur la période d'échantillonnage se situent entre $2,78 \pm 0,68 \mu \mathrm{g} / \mathrm{L}$ (Taboth) et 16,38 $\pm 13,59$ $\mu \mathrm{g} / \mathrm{L} \quad$ (Gbougbo) (Figure 2). Les concentrations en plomb fluctuent considérablement d'une station à une autre comme l'indiquent les valeurs élevées d'écart type. La distribution spatiale du plomb révèle des teneurs supérieures à la valeur de référence retenue par la Commission Européenne $(5 \mu \mathrm{g} / \mathrm{L})$. Il existe une différence significative selon l'analyse Anova $(\mathrm{P}<0,05)$ entre les stations pour la teneur en plomb. Pour l'ensemble des stations, le plomb apparaissait dans l'ordre : Gbougbo > Songon $>$ Koko > Bapoga > Ahua > Layo > Mopoyem $>$ N'Djem $>$ Abraco $>$ Papoga $>$ Taboth.

Les concentrations mensuelles du plomb pendant la période d'étude sont indiquées dans la Figure 3. Aucune tendance saisonnière n'est observée au cours de la période d'étude. Les concentrations saisonnières du plomb variaient de 1,35 à $42,80,1,35$ à 35,$45 ; 0,16$ à 9,45 et 0,16 à $35,45 \mu \mathrm{g} / \mathrm{L}$ avec une moyenne de $6,17 \pm 6,57$; $7,27 \pm 6,43 ; 3,40 \pm 2,94$ et $6,67 \pm 8,46 \mu \mathrm{g} / \mathrm{L}$ respectivement pendant la grande saison sèche (GSS), la grande saison des pluies (GSP), la petite saison sèche (PSS) et la petite saison des pluies (PSP). Le niveau de contamination suit l'ordre GSP $=$ PSP > GSS > PSS. La teneur la plus élevée a été obtenue pendant la fin de la grande saison sèche $(42,80 \mu \mathrm{g} / \mathrm{L}$, mars), tandis que les plus faibles ont été observées à la fin de la petite saison sèche (septembre) et au début de la petite saison des pluies (octobre). A Gbougbo, 67\% des valeurs enregistrées au cours de la campagne sont supérieures à la valeur normale du plomb dans les eaux naturelles. 
Les concentrations mensuelles du cadmium pendant la période d'étude sont indiquées sur la Figure 3. Aucune tendance n'est observée au cours de la période d'étude. Les concentrations saisonnières du cadmium ont varié de 0,3 à 31,$47 ; 0,5$ à 49,$59 ; 1,2$ à 31,2 et 0,76 à $40,15 \mu \mathrm{g} / \mathrm{L}$ avec une moyenne variant de $11,21 \pm 9,16 ; 11,82 \pm 11,04 ; 9,48 \pm$ 8,00 et $10,28 \pm 9,66 \mu \mathrm{g} / \mathrm{L}$ respectivement pendant la grande saison sèche, la grande saison des pluies, la petite saison sèche et la petite saison des pluies. Le niveau de contamination suit l'ordre GSP > GSS > PSP > PSS. La teneur la plus élevée a été obtenue pendant la grande saison de pluie $(50 \mu \mathrm{g} / \mathrm{L}$, juin), tandis que la plus faible a été observée pendant la grande saison sèche $(0,3 \mu \mathrm{g} / \mathrm{L}$, février).

Les concentrations moyennes du metalloïde arsenic varient entre $2,48 \pm 0,84$ $\mu \mathrm{g} / \mathrm{L}$ (Layo) et $23,86 \pm 37,45 \mu \mathrm{g} / \mathrm{L}$ (Ahua) (Figure 2). L'analyse de variance permet d'observer une différence significative entre les stations $(\mathrm{P}<0,05)$. L'ordre de contamination des eaux des stations en arsenic est Ahua $>$ Gbougbo $>$ Songon $>$ N'Djem $>$ Mopoyem > Bapo > Koko > Abraco > Taboth $>$ Layo > Papoga. Les concentrations mensuelles en arsenic pendant la période d'étude sont indiquées sur la Figure 3. Aucune tendance saisonnière n'a été observée au cours de la période d'étude. Les concentrations saisonnières en arsenic ont été comprises entre 0,49 et 53,$65 ; 0,93$ et 47,$83 ; 0,96$ et 87,40 et 1,59 et $96,31 \mu \mathrm{g} / \mathrm{L}$ avec une moyenne variant entre $6,22 \pm 9,92 ; 5,26 \pm 7,43 ; 12,12$ $\pm 21,96$ et $8,73 \pm 19,37 \mu \mathrm{g} / \mathrm{L}$ respectivement pendant la grande saison sèche, la grande saison des pluies, la petite saison sèche et la petite saison des pluies. Le niveau de contamination suit l'ordre PSS > PSP > GSS > GSP. La teneur la plus élevée a été obtenue au début de la petite saison de pluie $(96 \mu \mathrm{g} / \mathrm{L}$, octobre), tandis que la plus faible a été observée pendant la grande saison sèche $(0,3$ $\mu \mathrm{g} / \mathrm{L}$, février). L'analyse de la variation saisonnière de l'arsenic montre que, seulement $25 \%$ des valeurs dépassent la valeur normale de l'arsenic.

\section{Evaluation de la qualité des eaux lagunaire}

Les concentrations moyennes en arsenic, cadmium et plomb obtenues nous ont permis d'évaluer la qualité des eaux de la zone d'étude en les comparants aux valeurs limites chronique et aigüe préconisées pour la vie des organismes aquatiques (Tableau 4). Il ressort que la concentration moyenne d'arsenic $(7,30 \pm 14,14 \mu \mathrm{g} / \mathrm{L})$ est inférieure à la valeur chronique $(150 \mu \mathrm{g} / \mathrm{L})$ tandis que celle du plomb $(6,16 \pm 6,56 \mu \mathrm{g} / \mathrm{L})$ est supérieure à concentration chronique limite $(0,62 \mu \mathrm{g} / \mathrm{L})$. Contrairement à l'arsenic et au plomb, la concentration moyenne en cadmium $(\approx 11 \pm 9,77 \mu \mathrm{g} / \mathrm{L})$ a été supérieure à la concentration chronique $(0,10 \mu \mathrm{g} / \mathrm{L})$ et à la concentration aigüe $(0,58 \mu \mathrm{g} / \mathrm{L})$ pour la vie aquatique.

\section{Évaluation des risques pour la santé humaine}

Les doses journalières d'exposition par voie cutanée (DJE) sont présentées dans le Tableau5. Les valeurs de DJE ont été comprises entre 0,0001 et $0,0166 \mu \mathrm{g} / \mathrm{kg} / \mathrm{j}$ pour l'arsenic, entre 0,0001 et $0,0085 \mu \mathrm{g} / \mathrm{kg} / \mathrm{j}$ pour le cadmium et entre 0,0001 et $0,0294 \mu \mathrm{g} / \mathrm{kg} / \mathrm{j}$ pour le plomb. Les valeurs moyennes de DJE ont été de $0,0041 \pm 0,0064 \mu \mathrm{g} / \mathrm{kg} / \mathrm{j} ;$ de 0,0041 $\pm 0,0022 \mu \mathrm{g} / \mathrm{kg} / \mathrm{j} ;$ de $0,0113 \pm 0,0093 \mu \mathrm{g} / \mathrm{kg} / \mathrm{j}$ pour l'arsenic, le cadmium et le plomb, respectivement. Les doses d'ingestion par voie cutanée de l'arsenic et cadmium ont été inférieures aux doses de références qui sont de $0,009 \mu \mathrm{g} / \mathrm{kg} / \mathrm{j}$ pour l'arsenic et de 0,0005 $\mu \mathrm{g} / \mathrm{kg} / \mathrm{j}$ pour le cadmium (USEPA, 2004 ; US DoE, 2005). Par contre, la dose d'ingestion du plomb a été sensiblement égale à la dose de référence $(0,0014 \mu \mathrm{g} / \mathrm{kg} / \mathrm{j})$ (USEPA, 2004 ; USDoE, 2005).

Les valeurs des indices non cancérigènes (HQ) et de risque (HI) sont présentées aux Figures 4 et 5 . Ces valeurs de HQ et HI sont inférieures à 1 pour l'ensemble des stations étudiées. Ce résultat suggère que l'arsenic, le cadmium et le plomb dans les eaux de lagune ne présentent aucun risque non cancérigène. 


\section{A. M. O. TOGBE et al. / Int. J. Biol. Chem. Sci. 13(2): 1162-1179, 2019}

Tableau 3 : Concentration des eaux de surface $(\mu \mathrm{g} / \mathrm{L})$ en $\mathrm{Pb}, \mathrm{Cd}$ et As.

\begin{tabular}{|c|c|c|c|c|c|c|c|c|c|c|c|c|c|c|}
\hline & & \\
\hline & & Fév & Mar & Avr & Mai & Jui & Juil & Août & Sept. & Oct & Nov & Déc & Janv & Moy \\
\hline \multirow{10}{*}{$\mathrm{Pb}$} & AHUA & 2,04 & 3,86 & 2,14 & 7,66 & 7,68 & 7,49 & 8,38 & 9,45 & 18,76 & 2,54 & 2,74 & 2,39 & 6,26 \\
\hline & KOKO & 4,78 & 3,42 & 7,32 & 2,48 & 2,52 & 2,36 & 7,56 & 7,37 & 7,57 & 13,81 & 8,51 & 13,14 & 6,74 \\
\hline & TABOTH & 2,56 & 4,26 & 3,48 & 2,7 & 2,64 & 2,82 & 2,32 & 1,95 & 1,82 & 3,48 & 2,7 & 2,64 & 2,78 \\
\hline & N'DJEM & 5,16 & 3,4 & 4,27 & 7,37 & 4,71 & 5,78 & 0,8 & 0,57 & 0,78 & 4,27 & 7,37 & 4,71 & 4,10 \\
\hline & SONGON & 12,32 & 11,72 & 12,26 & 10,66 & 10,06 & 10,64 & 0,18 & 0,16 & 0,16 & 22,58 & 10,66 & 10,06 & 9,29 \\
\hline & LAYO & 3,71 & 5,5 & 4,37 & 4,79 & 7,76 & 6,64 & 3,29 & 2,51 & 2,9 & 4,37 & 4,79 & 7,76 & 4,87 \\
\hline & MOPOYE & 3,28 & 1,39 & 2,49 & 5,62 & 11,16 & 5,47 & 0,69 & 2,02 & 0,51 & 2,49 & 5,62 & 11,16 & 4,33 \\
\hline & ABRACO & 6,32 & 4,26 & 5,12 & 2,48 & 2,39 & 2,49 & 1,3 & 2,78 & 2,36 & 5,12 & 2,48 & 2,39 & 3,29 \\
\hline & GBOUBO & 2,6 & 42,8 & 35,45 & 14,51 & 10,32 & 12,08 & 7,21 & 6,49 & 4,78 & 35,45 & 14,51 & 10,32 & 16,38 \\
\hline & Moy. & 4,55 & 7,92 & 7,47 & 6,8 & 7,52 & 7,28 & 3,31 & 3,49 & 4,21 & 9,13 & 5,76 & 6,45 & 6,16 \\
\hline \multirow[t]{4}{*}{$\mathrm{Cd}$} & TABOTH & 0,3 & 1,1 & 0,5 & 3,24 & 3,07 & 2,92 & 2,79 & 9,7 & 2,16 & 4,5 & 7,2 & 5,7 & 3,60 \\
\hline & N'DJEM & 16,5 & 11,2 & 18,2 & 5,8 & 1,4 & 1,07 & 1,93 & 3,5 & 1,97 & 14,76 & 22,93 & 13,99 & 9,44 \\
\hline & SONGON & 19 & 12,8 & 9,5 & 20 & 15,83 & 18,47 & 2,57 & 11,8 & 2,08 & 2,08 & 6,5 & 10,3 & 10,91 \\
\hline & PAPOGA & 0,8 & 1,4 & 0,8 & 11,2 & 9,83 & 21,8 & 7,65 & 6,8 & 7,36 & 18,86 & 27,05 & 21,71 & 11,27 \\
\hline
\end{tabular}


A. M. O. TOGBE et al. / Int. J. Biol. Chem. Sci. 13(2): 1162-1179, 2019

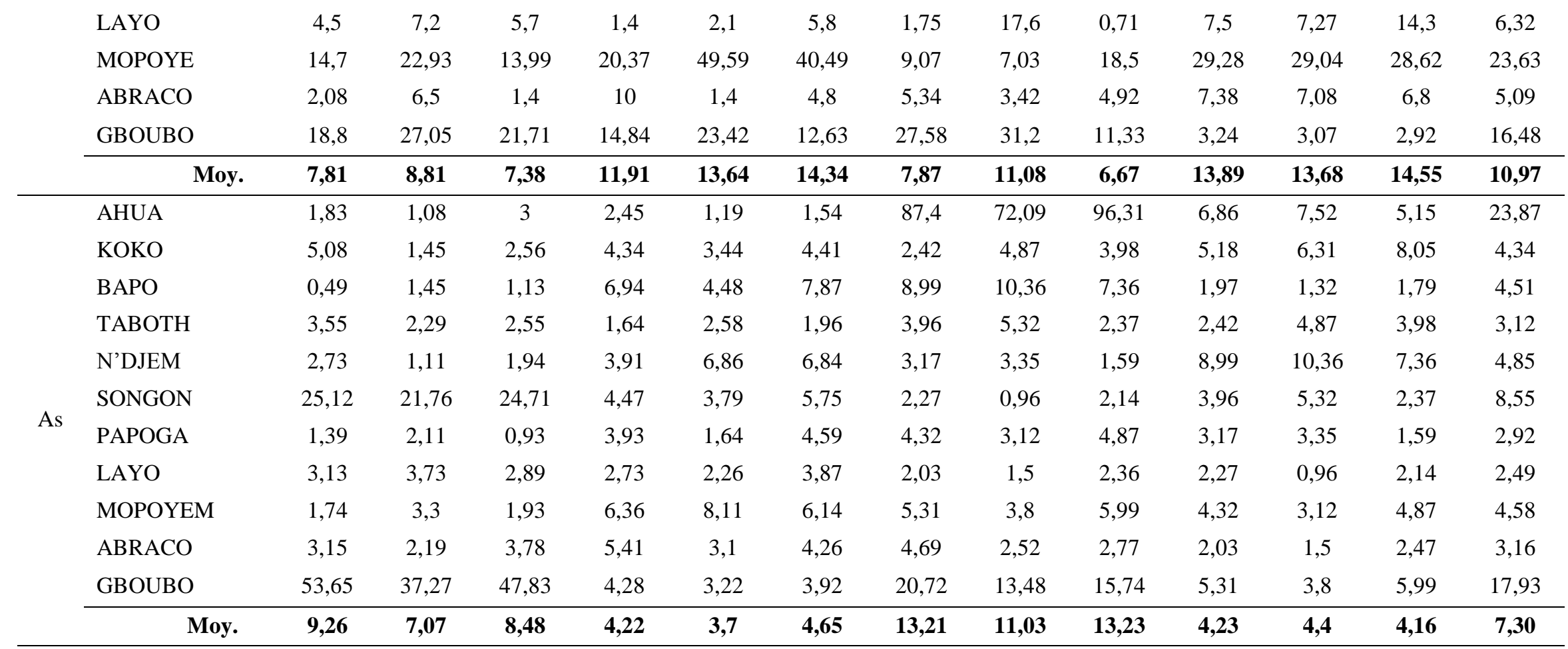



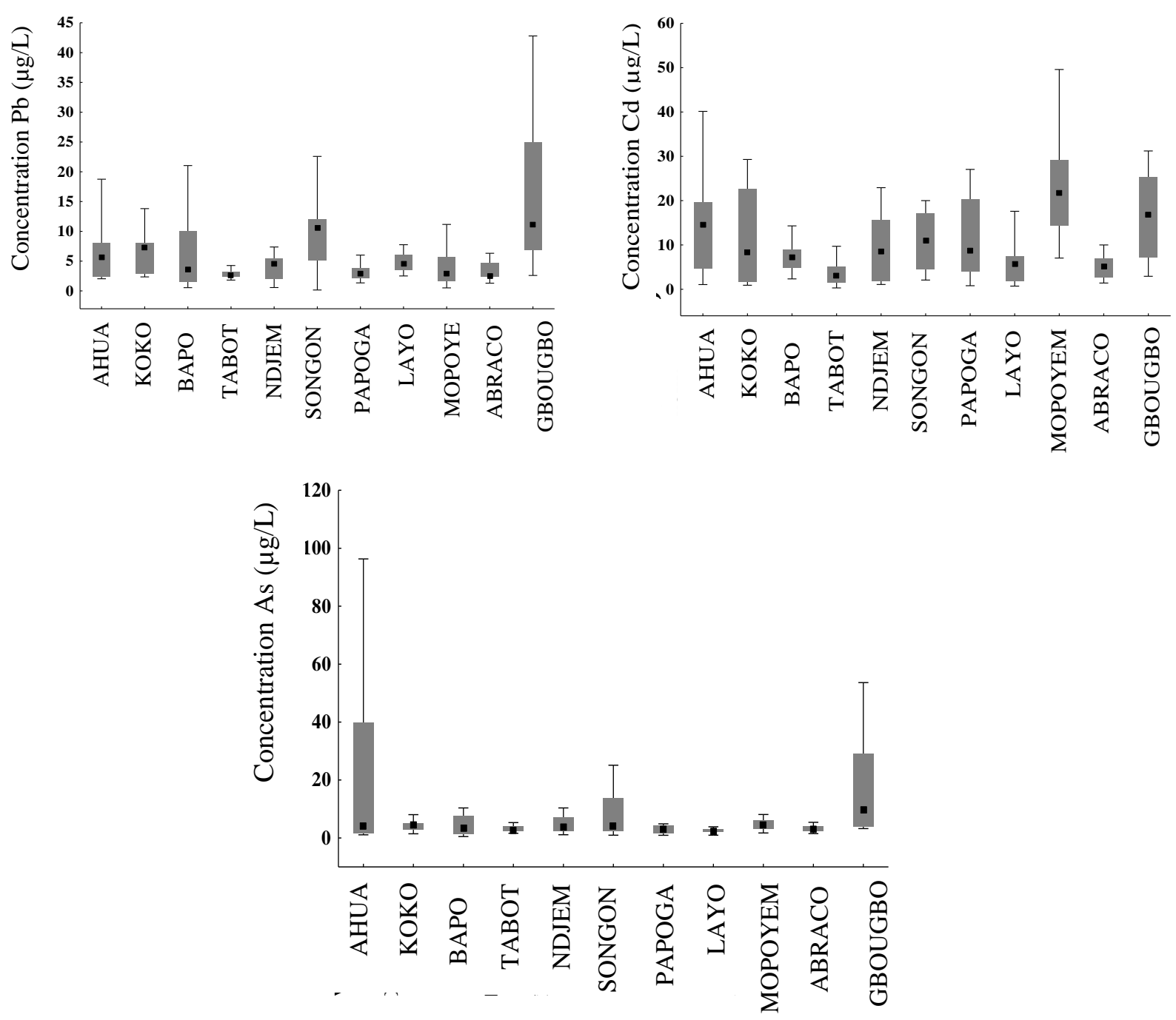

Figure 2 : Variation spatiale de la concentration des métaux plomb, cadmium et arsenic dans les eaux de la lagune.

Tableau 4 : Comparaison des valeurs de $\mathrm{Pb}, \mathrm{Cd}$ et As avec les critères de protection de la vie aquatique selon le MDDEP (2006).

\begin{tabular}{lllcc}
\hline Paramètres & unités & $\begin{array}{l}\text { Valeurs } \\
\text { mesurées dans } \\
\text { cette étude }\end{array}$ & $\begin{array}{c}\text { Protection de la vie aquatique* } \\
\text { Aigüe }\end{array}$ & Chronique \\
& & & & \\
\hline Métaux & 7 & 340 & 150 \\
Arsenic & $\mu \mathrm{g} / \mathrm{L}$ & 11 & 0,58 & 0,10 \\
Cadmium & $\mu \mathrm{g} / \mathrm{L}$ & 6 & 16 & 0,62 \\
Plomb & $\mu \mathrm{g} / \mathrm{L}$ & 6 & & \\
\hline
\end{tabular}


A. M. O. TOGBE et al. / Int. J. Biol. Chem. Sci. 13(2): 1162-1179, 2019

Tableau 5 : Statistique des valeurs de la DJE (Dose Journalière d'Exposition par voie cutanée).

\begin{tabular}{|c|c|c|c|c|c|c|c|c|c|c|c|c|}
\hline \multirow[t]{2}{*}{ DJE } & \multicolumn{4}{|c|}{$\mathbf{P b}$} & \multicolumn{4}{|c|}{ Cd } & \multicolumn{4}{|c|}{ As } \\
\hline & Min & Max & Moy & SD & Min & Max & Moy & SD & Min & Max & Moy & SD \\
\hline AHUA & 0,0014 & 0,0129 & 0,0043 & 0,0033 & 0,0002 & 0,0069 & 0,0025 & 0,0021 & 0,0002 & 0,0166 & 0,0041 & 0,0064 \\
\hline KOKO & 0,0016 & 0,0095 & 0,0046 & 0,0027 & 0,0002 & 0,0050 & 0,0021 & 0,0020 & 0,0002 & 0,0014 & 0,0007 & 0,0003 \\
\hline BAPO & 0,0004 & 0,0145 & 0,0046 & 0,0053 & 0,0004 & 0,0025 & 0,0013 & 0,0006 & 0,0001 & 0,0018 & 0,0008 & 0,0006 \\
\hline TABOTH & 0,0013 & 0,0029 & 0,0019 & 0,0005 & 0,0001 & 0,0017 & 0,0006 & 0,0005 & 0,0003 & 0,0009 & 0,0005 & 0,0002 \\
\hline N'DJEM & 0,0004 & 0,0051 & 0,0028 & 0,0016 & 0,0002 & 0,0039 & 0,0016 & 0,0013 & 0,0002 & 0,0018 & 0,0008 & 0,0005 \\
\hline SONGON & 0,0001 & 0,0155 & 0,0064 & 0,0044 & 0,0004 & 0,0034 & 0,0019 & 0,0011 & 0,0002 & 0,0043 & 0,0015 & 0,0016 \\
\hline PAPOGA & 0,0009 & 0,0041 & 0,0021 & 0,0009 & 0,0001 & 0,0047 & 0,0019 & 0,0016 & 0,0002 & 0,0008 & 0,0005 & 0,0002 \\
\hline LAYO & 0,0017 & 0,0053 & 0,0033 & 0,0012 & 0,0001 & 0,0030 & 0,0011 & 0,0009 & 0,0002 & 0,0007 & 0,0004 & 0,0001 \\
\hline MOPOYEM & 0,0004 & 0,0077 & 0,0030 & 0,0025 & 0,0012 & 0,0085 & 0,0041 & 0,0022 & 0,0003 & 0,0014 & 0,0008 & 0,0003 \\
\hline ABRACO & 0,0009 & 0,0043 & 0,0023 & 0,0010 & 0,0002 & 0,0017 & 0,0009 & 0,0005 & 0,0003 & 0,0009 & 0,0005 & 0,0002 \\
\hline GBOUBO & 0,0018 & 0,0294 & 0,0113 & 0,0093 & 0,0005 & 0,0054 & 0,0028 & 0,0017 & 0,0006 & 0,0092 & 0,0031 & 0,0031 \\
\hline
\end{tabular}



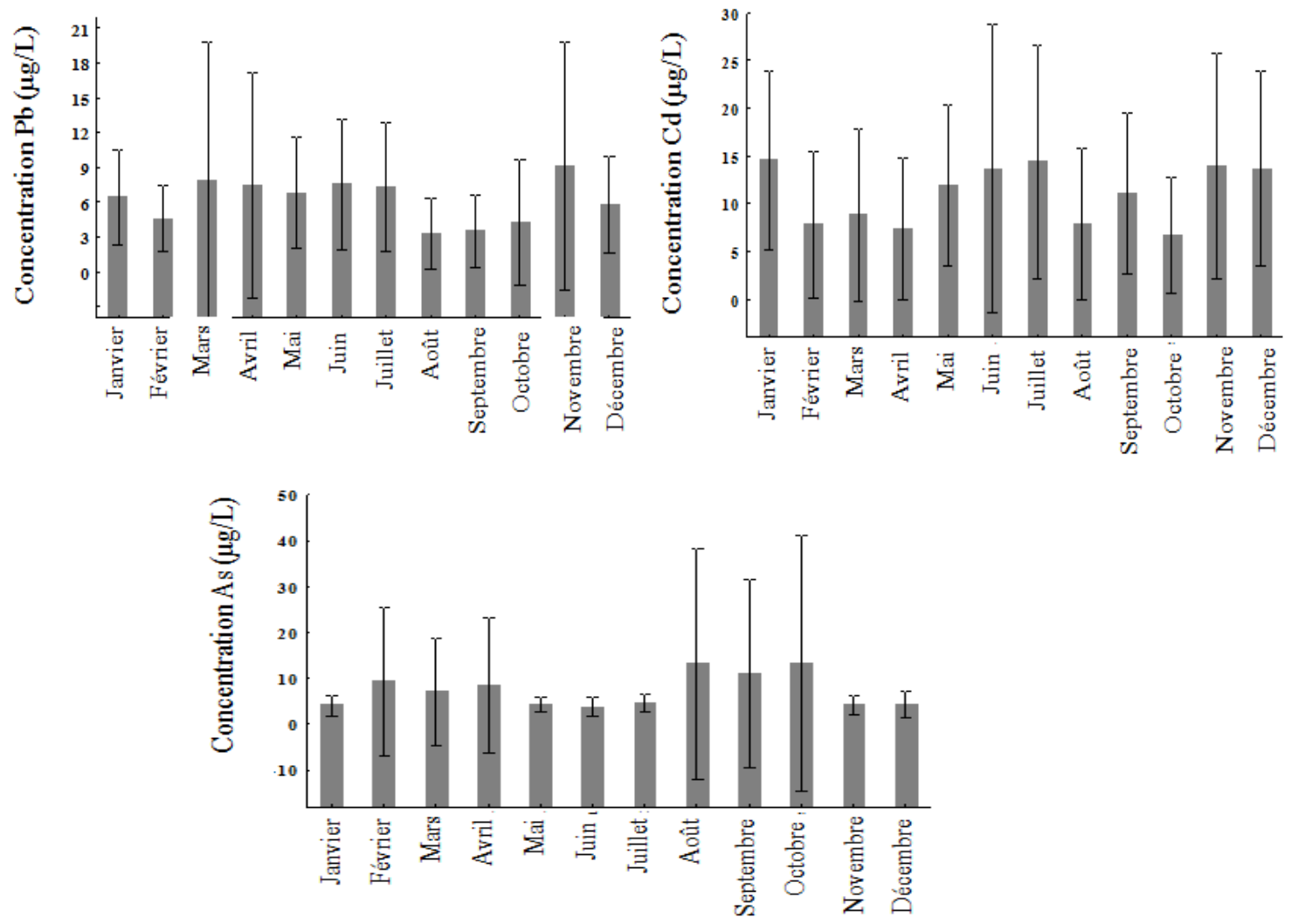

Figure 3 : Variation saisonnière de la concentration des métaux plomb, cadmium et arsenic dans les eaux de la lagune.

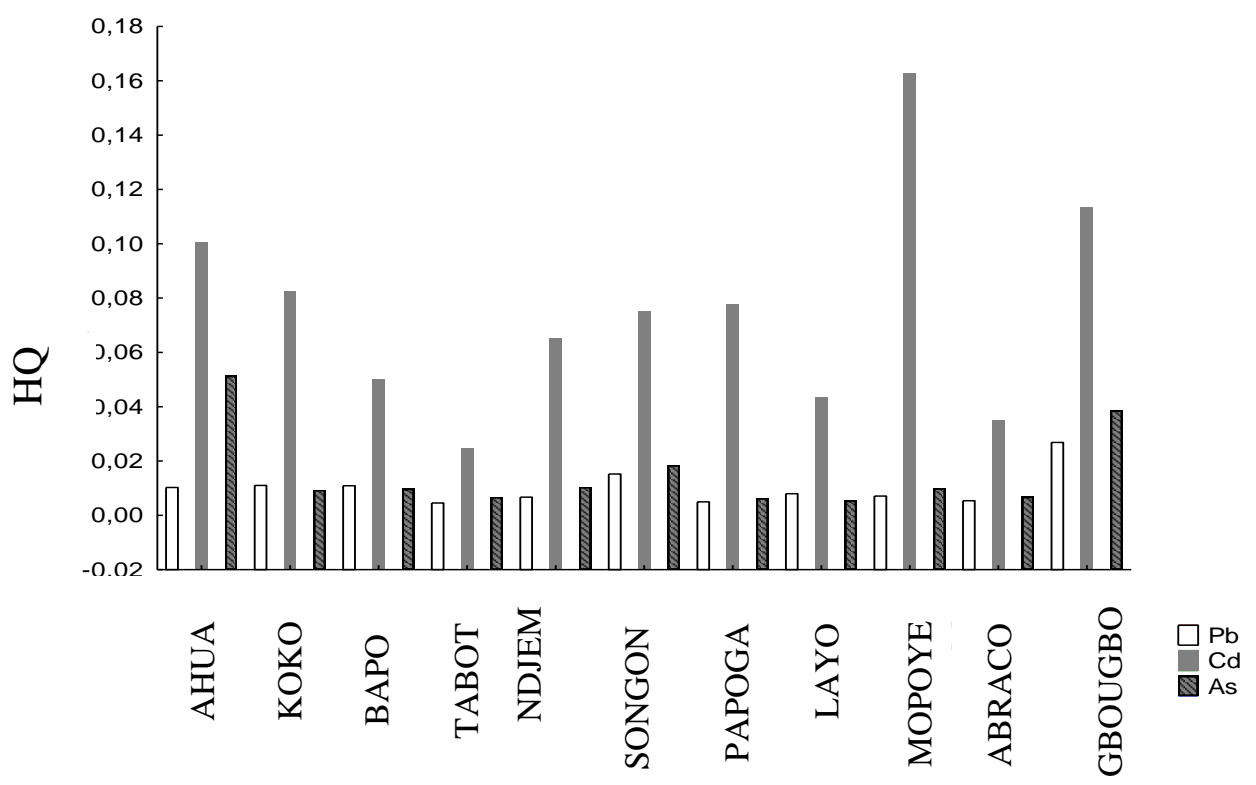

Figure 4 : Risques non cancérigènes(HQ) des eaux lagunaires. 


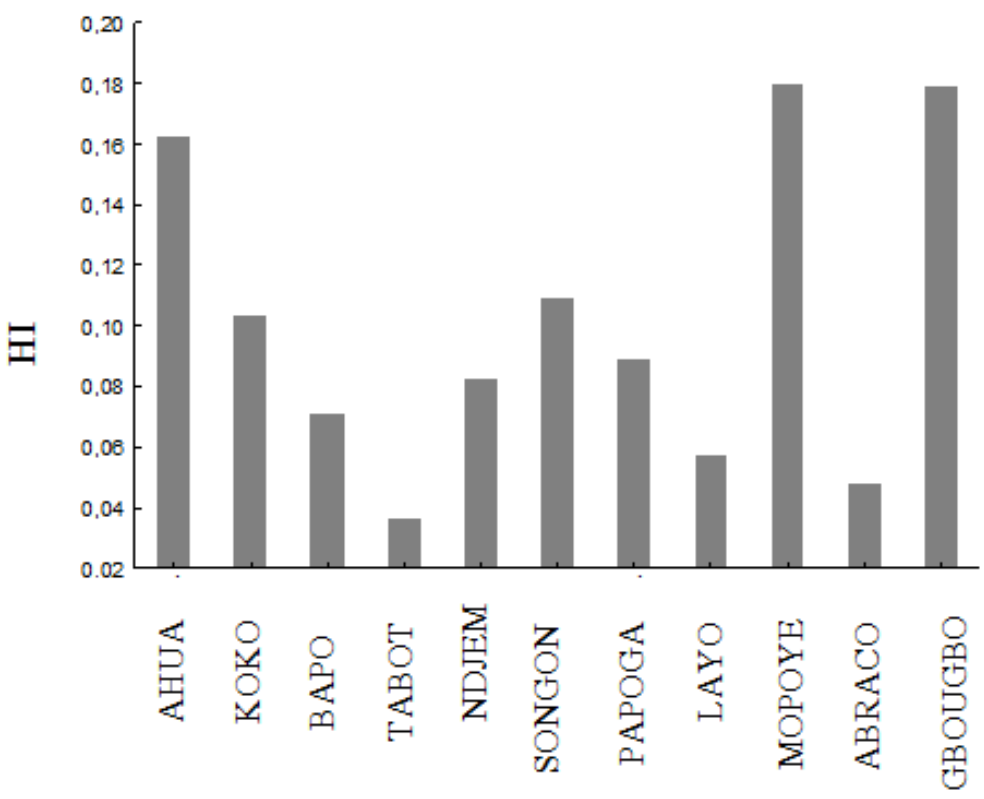

Figure 5 : Indice de risques non cancérigènes(HI) des eaux lagunaires.

\section{DISCUSSION}

Les concentrations en arsenic ont été comprises entre $(0,49-96,31 \mu \mathrm{g} / \mathrm{L})$ avec une moyenne de $7,30 \pm 14,14 \mu \mathrm{g} / \mathrm{L}$ pour l'ensemble des stations, se sont avérées faibles comparativement aux concentrations chronique $(150 \mu \mathrm{g} / \mathrm{L})$ et aigüe $(340 \mu \mathrm{g} / \mathrm{L})$ de protection de la vie aquatique (MDDEP, 2006). Toutefois, les fortes concentrations en arsenic obtenues à Ahua $(87,4-76,31 \mu \mathrm{g} / \mathrm{L})$ pourraient s'expliquer par le développement des activités agricoles et l'urbanisation grandissante dans la zone. Comparativement aux concentrations en arsenic $(0,16-5,89$ $\mu \mathrm{g} / \mathrm{L}$ ) rapportées par Wang et al. (2018) de l'estuaire de Yellow River, en Chine, celles trouvés dans cette étude se sont montrées supérieures. De même, Li et al. (2017) ont rapporté des concentrations en arsenic $(3,8-$ $11,3 \mu \mathrm{g} / \mathrm{L}$ ) dans l'estuaire Yalujiang, en Chine inférieures à celles obtenues par la présente étude. La variation saisonnière a montré des concentrations en arsenic moyennes allant de $6,22 \pm 9,92$ à $12,12 \pm 21,96 \mu \mathrm{g} / \mathrm{L}$. Ce résultat montre une faible variation de la concentration en arsenic au cours des différentes saisons. L'analyse de variance (ANOVA) n'a montré aucune différence significative $(\mathrm{p}<0,05)$ pour les quatre saisons.

Les concentrations en cadmium ont varié de 0,3 à $40,15 \mu \mathrm{g} / \mathrm{L}$ avec une moyenne de $10,97 \pm 9,77 \mu \mathrm{g} / \mathrm{L}$ pour l'ensemble des stations. A l'exception de la plus faible valeur $(0,3 \mathrm{~g} / \mathrm{L})$, toutes les concentrations de cadmium obtenues ont été supérieures à la valeur limite $(0,58 \mu \mathrm{g} / \mathrm{L})$ (concentration aigüe) de protection de la vie aquatique préconisée. Ce résultat suggère que le cadmium présente un risque important pour les organismes aquatiques tels que les poissons. Ces valeurs élevées de cadmium dans les eaux de la lagune (secteurs IV et V) pourraient s'expliquer par les effluents provenant des unités agroindustrielles qui se déversent dans la lagune. En outre le drainage des résidus d'engrais utilisés dans les plantations industrielles (cocoterais et d'hévéa) situées dans la zone d'étude contribuerait à augmenter la concentration de cadmium dans les eaux de lagune. En effet, Harley et al. (2013) ont montré que plus de 54 à $58 \%$ de la concentration de cadmium trouvé dans les sols agricoles proviendrait des fertilisants phosphatés utilisés. La concentration de cadmium $(0,99 \mu \mathrm{g} / \mathrm{L})$ 
rapportée par Kouassi et al. (2015) dans les secteurs (I, II et III) de la Lagune Ebrié est inférieure à celles obtenues dans la présente étude.

La variation saisonnière de la concentration du cadmium a montré des valeurs moyennes oscillant entre 9,48 $\pm 8,00$ et $11,82 \pm 11,04 \mu \mathrm{g} / \mathrm{L}$. La concentration de cadmium a varié très peu dans les différentes saisons. L'analyse de variance $(\mathrm{p}<0,05)$ n'a montré aucune différence significative entre la concentration de cadmium pour les quatre saisons climatiques.

Quant au plomb, les concentrations ont varié de $0,16 \mu \mathrm{g} / \mathrm{L}$ à $35,45 \mu \mathrm{g} / \mathrm{L}$, avec une moyenne de $6,16 \pm 6,57 \mu \mathrm{g} / \mathrm{L}$ pour l'ensemble des stations. Ce résultat montre que la concentration moyenne en cadmium des eaux de lagune de la zone étudiée est 10 fois supérieure à la valeur seuil de la concentration chronique $(0,62 \mu \mathrm{g} / \mathrm{L})$ de plomb pour la protection de la vie aquatique. Ces concentrations élevées en plomb pourraient être dues à forte pression anthropique (activités agricoles, tourisme, industries agroindustrielles...) subit par la ville de Jacqueville où se situe la zone d'étude. Par ailleurs, la présence de plateformes de dragage de sable à la station de Koko pourrait également justifier les concentrations élevées de plomb observées. Praveena et al. (2008) ont également rapporté de fortes concentrations en plomb dans eaux de lagunes proches des habitations et des zones industrielles. Comparativement aux concentrations de plomb rapportées en Australie (Hatje et al., 2003), en Chine (Li et al., 2017 ; Wang et al., 2018), celles de la présente étude se sont avérées supérieures.

$\mathrm{La}$ variation saisonnière de la concentration en plomb a montré des valeurs moyennes allant de $3,40 \pm 2,94 \mu \mathrm{g} / \mathrm{L}$ à 7,27 $\mu \mathrm{g} / \mathrm{L}$ pour les différentes saisons. Il ressort que la concentration en plomb varie faiblement d'une saison à l'autre. L'analyse de variance n'a montré aucune différence significative $(\mathrm{p}<0,05)$ entre les concentrations de plomb des quatre saisons.

\section{Evaluation de la contamination des eaux lagunaires}

La qualité des eaux de lagune dans la zone d'étude a été comparée aux critères de vie aquatique canadienne. A l'exception de l'arsenic, les concentrations de cadmium et de plomb constituent un danger pour les organismes aquatiques dans les secteurs IV et $\mathrm{V}$ de la lagune Ebrié. Ces résultats sont en accord avec ceux de Bakary et al. (2015) qui ont rapporté de fortes concentrations en $\mathrm{Cd}$ et $\mathrm{Pb}$ dans les eaux de la lagune Ebrié (Baie des Milliardaires).

\section{Risques sanitaires}

Les valeurs moyennes des doses journalières ingérées par voie cutanée (DJE) montrent que les doses d'arsenic, de cadmium et de plomb ne présentent pas de risque pour la santé humaine. Toutefois, les valeurs maximales des DJE enregistrées à Gbougbo $(0,0294 \mu \mathrm{g} / \mathrm{kg} / \mathrm{j})$, à Mopoyem $(0,085 \mu \mathrm{g} / \mathrm{kg} / \mathrm{j})$ et à Ahua $(0,0166 \mu \mathrm{g} / \mathrm{kg} / \mathrm{j})$ pour le plomb, le cadmium et l'arsenic respectivement pourraient constituer un danger pour la population. En effet, la zone d'étude (Jacqueville) est prisée par les populations pour la baignade.

Les valeurs des indices $\mathrm{HQ}$ et $\mathrm{HI}$ inférieures à 1 montrent que les trois éléments étudiés ne présentent pas de risque non cancérigène pour la population dans la zone d'étude.

\section{Conclusion}

Dans cette étude, les niveaux de contamination des eaux lagunaires en plomb, en cadmium et en arsenic ont été évalués. Les variations spatiotemporelles, la qualité des eaux pour la vie aquatiques, de même que les risques potentiels pour les populations exposées ont été examinés. L'étude de la variation spatiale des éléments traces métalliques étudiés a montré que la plupart des concentrations en cadmium et en plomb sont supérieures à celle des autres stations surtout pendant la saison des pluies. Cependant, l'analyse de variance n'a montré aucune différence significative entre la 
concentration des métaux dans les quatre saisons. A l'exception de l'arsenic, les concentrations en cadmium et en plomb dans les eaux ont été supérieures à la concentration chronique pour la vie des organismes aquatiques, selon les critères canadiens. L'évaluation des risques pour la santé indique que les quantités d'absorption journalière (DJE) maximales par voie cutanée des éléments $(\mathrm{As}, \mathrm{Cd}$ et $\mathrm{Pb})$ pourraient constituer une menace pour la santé des populations locales. Cependant, les valeurs des indices HQ et $\mathrm{HI}$ inférieures à 1 montrent que les concentrations de métaux ne présentent pas de risques non cancérigènes. Contrairement à la plupart des travaux qui se sont focalisés sur les secteurs I, II et III, cette étude constitue une première dans les secteurs IV et $\mathrm{V}$ de la lagune Ebrié. Par ailleurs, le développement des activités économiques dans cette zone pourrait impacter davantage la qualité des eaux de la lagune. Par conséquent, une collecte continue et/ou périodique de données sur la qualité des eaux des secteurs IV et $\mathrm{V}$ de la lagune Ebrié s'avère essentielle pour préserver la santé des populations.

\section{CONFLIT D'INTERETS}

Les auteurs déclarent n'avoir aucun conflit d'intérêts.

\section{CONTRIBUTION DES AUTEURS}

La contribution des auteurs à la réalisation des travaux de laboratoire, à la rédaction et aux différentes corrections s'est établie comme suit: AMOT a été l'instigateur et le rédacteur principal de cet article et a effectué les travaux de collecte et de laboratoire. KVK a contribué par ses critiques avisées et conseils pour la rédaction du manuscrit. KMY a conçu et supervisé le travail. Il a aussi été l'un des rédacteurs de ce manuscrit. $\mathrm{OHH}$ a aidé au traitement des données. AST a été la directrice scientifique de ce travail et BCA en a été le directeur technique.

\section{REMERCIEMENTS}

Le présent travail a été effectué dans le cadre des effets des changements environnementaux des milieux lagunaires sur l'Aquaculture continentale et la pêche artisanale dans la portion occidentale de la lagune Ebrié en Côte d'Ivoire. Le fonds Interprofessionnel pour la Recherche et le Conseil Agricole (FIRCA) a assuré le financement de la présente étude. Les professeurs Atsé Bouah et Tidou Abiba Sanogo ont assuré la direction scientifique du projet Les auteurs expriment leur gratitude à l'ensemble des partenaires impliqués dans toutes les opérations afférentes à la collecte et aux traitements des échantillons (eaux, poissons et sédiments) :

Drs Nobah Céline Sidonie Koco (ENS), Coulibaly safiatou (CRO), Yao Konan Marcel (UFHB), Kouamé Victor (UNA), Mrs Koné Kanakounou jean-marie (CRO), Boni Juste Géraud (CIAPOL), N'guessan Kouadio Rigobert (CIAPOL), Kinimo Charles (Université de Korhogo) Assi Yapo Joseph (CRO), Kouakou Kouadio (CRO), Bopo Zadi Sylvain Olivier (CRO), Kouamelan N'zébo Moîse (UFHB) and Ms Boni laurence (UFHB).

\section{REFERENCES}

Adama DT, Metongo BS, Trokourey A, Bokra Y. 2012. Assessment of Waters Contamination by Nutrients and Heavy Metals in the Ebrie Lagoon (Abidjan, Ivory Coast). Research Journal of Environmental Toxicology, 6: 198-209. DOI: 10.3923/rjet.2012.198.209.

Ahmad K, Bhatti IA, Muneer M, Iqbal M, Iqbal Z. 2012. Removal of heavy metals $(\mathrm{Zn}, \mathrm{Cr}, \mathrm{Pb}, \mathrm{Cd}, \mathrm{Cu}$ and $\mathrm{Fe}$ ) in aqueous media by calcium carbonate as an adsorbent. International Journal of Chemical and Biochemical Sciences, 2: 48-53. DOI: 10.1.1.718.3855

Ahmad MK, Islam S, Rahman MS, Haque MR, Islam MM. 2010. Heavy Metals in Water, Sediment and Some Fishes of Buriganga River, Bangladesh. Int. J. 
Environ. $\quad$ Res., $\quad \mathbf{4}(2): 321-332$. DOI: $10.1007 / \mathrm{s} 11356-019-04495-6$

An Q, Wu Y, Wang J, Li Z .2010. Assessment of dissolved heavy metal in the Yangtze River estuary and its adjacent sea, China. Environ. Monit. Assess., 164(1-4): 17387. DOI: $10.1007 / \mathrm{s} 10661-009-0883-\mathrm{z}$

APHA (American Public Health Association) 1998. Standard Methods for the Examination of Water and Wastewater. 20th Edition APHA: Washington DC.

Babarinde A, Babalola JO, Adegoke J, Osundeko AO, Olasehinde S, Omodehin Adetayo, Nurhe E. 2013. Biosorption of $\mathrm{Ni}$ (II), $\mathrm{Cr}$ (III), and Co (II) from Solutions Using Acalypha hispida Leaf: Kinetics, Equilibrium, and Thermodynamics. Journal of Chemistry, (2013): 1-8. DOI:10.1155/2013/460635

Beliles RP. 1994. The metals. In: Patty's Idustrial Hygiene and Toxicology, Fourth edition. Clayton GD, Clayton FE (Ed), Volume 2, Part C. John Willey \& Sons, Inc.

Burgos-Nunez S, Navarro-Frometa A, Marrugo-Negrete J, Enamorado-Montes G, Urango-Cardenas I. 2017. Polycyclic aromatic hydrocarbons and heavy metals in the Cispata Bay, Colombia: a marine tropical ecosystem. Mar. Pollut. Bull., 120(1-2): $\quad 379-386 . \quad$ DOI: 10.1016/j.marpolbul.2017.05.016

Cheraghi M, Lorestani B, Merrikhpour $\mathrm{H}$. 2012. Investigation of the effects of phosphate fertilizer application on the heavy metal content in agricultural soils with different cultivation patterns. Biol. Trace Element Res., 145(1): 87-92. DOI: 10.1007/s12011-011-9161-3

Das P, Samantaray S, Rout R. 1997. Studies on cadmium toxicity in plants: a review. Environ Pollut., 98 (1): 29-36.

Dufour P, Kouassi AM, Lamusse A. 1994. Les pollutions: Environnement et Ressources Aquatiques de Côte d'Ivoire, Vol II. Durand JR, Dufour P, Guiral D, Zahi SGF (Eds). Editions de l'ORSTROM : Paris.
Durand JR, Guiral D. 1994. Hydroclimat et hydrochimie. In: Environnement et ressources aquatiques de Côte d'Ivoire. Les milieux lagunaires. ORSTOM, 2 : 59-90.

Foxtrot. 2012. Etude d'impact environnemental et social. Rapport final Foxtrot, 20-93.

Hartley TN, Macdonald AJ, McGrath SP, Zhao FJ, 2013. Historical arsenic contamination of soil due to long-term phosphate fertilizer applications. Environmental Pollution, 180 : 259-264.

Hatje V, Apte SC, Hales LT, Birch GF. 2003. Dissolved trace metal distributions in Port Jackson estuary (Sydney Harbour), Australia. Mar. Pollut. Bull., 46(6): 71930.

Hooda PS. 2010. Front Matter. In: Trace Elements in Soils, Hooda P. (Ed.). John Wiley and Sons, Ltd.: New York, USA.

Hu C, Yang X, Dong J, Zhang X. 2018. Heavy metal concentrations and chemical fractions in sediment from Swan Lagoon, China: Their relation to the physiochemical properties of sediment. J. Chemosphere, 1-32. DOI: 10.1016/j.chemosphere.2018.06.113.

Hyder M, Zafer MN, Gilani SR, Shaheer M. 2018. Concentration trends of $\mathrm{Pb}, \mathrm{Cr}$, $\mathrm{Cd}$, and $\mathrm{Hg}$ in fish from different habitats in Pakistan. International Journal of Chemical and Biochemical Sciences, 13(2018): 6-12.

Kinimo KC, Yao, KM, Marcotte S, Kouassi NLB, Trokourey A. 2018. Distribution trends and ecological risks of arsenic and trace metals in wetland sediments around gold mining activities in central-southern and southeastern Côte d'Ivoire. Journal of Geochemical Exploration, 190: 265280. DOI: 10.1016/j.gexplo.2018.03.013

Kouassi, NLB, Yao KM, Trokourey A, Soro B. 2015. Distribution, Sources, and Possible Adverse Biological Effects of Trace Metals in Surface Sediments of a Tropical Estuary. Environmental 
Forensics, $\quad 16$ : 96-108. DOI: 10.1080/15275922.2014.991433.

La Rocca B, Houeix N, Andres S. 2010. Arsenic et ses dérivés, Fiche de données toxicologiques et environnementales des substances chimiques. INERIS, 124p.

Li H, Lin L, Ye S, Li H, Fan J. 2017. Assessment of nutrient and heavy metal contamination in the seawater and sediment of Yalujiang Estuary. Mar. Pollut. Bull., 117(1-2): 499-506. DOI: 10.1016/j.marpolbul.2017.01.069.

Ouattara AA, Yao KM, Soro MP, Diaco T, Trokourey A. 2018. Arsenic and Trace Metals in Three West African rivers: Concentrations, Partitioning, and Distribution in Particle-Size Fractions. Archives of Environmental Contamination and Toxicology, 1-15. DOI: $10.1007 / \mathrm{s} 00244-018-0543-9$

FOXTROT, 2012. Etude d'impact environnemental et social. Rapport final FOXTROT, 20-93.

Perera PACT, Kodithuwakku S, Sundarabarathy T, Edirisinghe U. 2015. Bioaccumulation of Cadmium in Freshwater Fish: An Environmental Perspective. Insight Ecology., 4: 1-12. DOI: 10.5567/ECOLOGY-IK.2015.1.12

Praveena SM, Aris AZ, Radojevic M. 2010. Heavy metals and source in intertidal mangrove sediment of Sabah Borneo. Island Environment Asia, (3): 79-83.

Rauch JN, Pacyna JM. 2009. Earth's global $\mathrm{Ag}, \mathrm{Al}, \mathrm{Cr}, \mathrm{Cu}, \mathrm{Fe}, \mathrm{Ni}, \mathrm{Pb}$, and $\mathrm{Zn}$ cycles. Global Biogeochemical cycles, 23(2): 1-16. DOI: 10.1029/2008GB003376.

Sangaré N, Yao KM, Kwa-Koffi EK, Kouassi NLB, Soro MB, Kouassi AM. 2016. Évaluation de la qualité des ressources en eau près de la décharge urbaine non contrôlée d'Akouédo par le calcul des risques cancérigènes et des indices de pollution, Côte d'Ivoire. Afrique Science, 12(5): 279-290.

Sundaray SK, Nayakb BB, Lin S, Bhattac D. 2011. Geochemical speciation and risk assessment of heavy metals in the river estuarine sediments: A case study: Mahanadi basin, India. Journal of Hazardous Materials, 186(2-3): 18371846 .

DOI: 10.1016/j.jhazmat.2010.12.081.

Tuo AD, Yao KM, Soro MB, Trokourey A, Bokra Y.2013. Contamination of surface sediments by heavy metals in Ebrie Lagoon (Abidjan, Côte d'Ivoire). International Journal of Chemical Technology, 5(1): 10-21. DOI: 10.3923/ijct.2013.10.21.

UNEP (United nation for Environmental Programm) 2010. Final review of scientific information on cadmium. Chemicals Branch, DTIE. UNEP.

US EPA (US Environmental Protection Agency), 2013. Regional screening level (RSL) summary Table. US EPA. http://www.epa.gov/ reg3hwmd/risk/human/rb concentration_table/Generic_Tables/doc s/master_sl_table_run_NOV2013.pdf.

USDoE (US Departement of Energy), 2005. RAIs: risk assessment information system. USDoE. Available from: http://risk.isd.ornl.gov/rap_hp.shtml.

USEPA (US Environmental Protection Agency), 2004. Risk assessment guidance for superfund volume I: human health evaluation manual (Part E, supplemental guidance for dermal risk assessment). EPA/540/R/99/005. Office of superfund remediation and technology innovation US environmental protection agency. USEPA, Washington DC.

USEPA (US Environmental Protection Agency), 1990. National oil and hazardous substances pollution contingency plan, 40 CRF part 300. USEPA, Washington DC.

USEPA (US Environmental Protection Agency), 1991. Role of the baseline risk assessment in superfund remedy selection decisions Office of Solid Waste and Emergency Response. USEPA, OSWER directive 9355.0-30. 1991b, 11 $\mathrm{p}$ 
Wang X, Zhao L, Xu H, Zhang X. 2018 .Spatial and seasonal characteristics of dissolved heavy metals in the surface seawater of the Yellow River Estuary, China. Marine Pollution Bulletin, 137: 465-473.

DOI:

101016/j.marpollbull.2018.10.052

Wei X, Han L, Gao B, Zhou H, Lu J, Wan X. 2016. Distribution, bioavailability, and potential risk assessment of the metals in tributary sediments of Three Gorges Reservoir: The impact of water impoundment. Ecol. Indicat., 61: 667675. DOI: $\quad 10.1016 /$ j.ecolind. 2015.10.018.

World Bank 2016. The World Bank Group: Average Life Expectancy in Côte d'Ivoire in 2009. World Bank.
Yao KM, Métongo BS, Trokourey A, Bokra Y. 2009. Assessment of sediments contamination by heavy metals in a tropical lagoon urban area (Ebrié Lagoon, Côte d'Ivoire). European Journal of Scientific Research, 34(2): 280-289.

Zhang G, Bai J, Xiao R, Zhao Q, Jia J, Cui B, Liu X. 2017. Heavy metal fractions and ecological risk assessment in sediments from urban, rural and reclamationaffected rivers of the Pearl River Estuary, China. J. Chemosphere, (184): 278-288. DOI:

10.1016/j.chemospere.2017.05.155 